\title{
The effects of knee meniscectomy on the development of osteoarthritis in the patellofemoral joint 40 years following meniscectomy
}

\author{
Ioannis Pengas ${ }^{1} \cdot$ William Nash $^{2} \cdot$ Angelos Assiotis $^{3} \cdot$ Kendrick To $^{4} \cdot$ Wasim Khan $^{4} \cdot$ Michael McNicholas $^{5}$
}

Received: 21 April 2019 / Accepted: 20 June 2019 / Published online: 10 July 2019

(c) The Author(s) 2019

\begin{abstract}
Most knee osteoarthritis and meniscectomy studies focus on osteoarthritis in the tibiofemoral joint and ignore the patellofemoral joint. This study aims to assess the long-term effects of total meniscectomy on the patellofemoral joint. To our knowledge, this is the only study of osteoarthritis in the patellofemoral joint following meniscectomy that extends to a 40-year follow-up period. Twenty-two patients with osteoarthritis were evaluated at a mean of 40 years post-meniscectomy using standardised weight-bearing radiographs of the operated and non-operated knees. Patellofemoral joint osteoarthritis was diagnosed by the presence of osteophytes and joint space narrowing to less than $5 \mathrm{~mm}$. Kellgren and Lawrence scores were calculated from the radiographs. Patellofemoral joint osteoarthritis and tibiofemoral joint osteoarthritis were correlated with International Knee Documentation Committee scores and range of movement measurements. A significant difference was observed between the operated and non-operated knees in terms of patellofemoral joint osteophyte formation. There was a significant difference in tibiofemoral joint Kellgren and Lawrence scores, International Knee Documentation Committee scores and range of movement measurements between knees with lateral facet patellofemoral joint space of $<5 \mathrm{~mm}$ and $>5 \mathrm{~mm}$. This study shows an association between open total meniscectomy and patellofemoral joint osteoarthritis at 40 years following surgery. There was also an association between patellofemoral joint space narrowing in the lateral facet and tibiofemoral joint osteoarthritis. Possible causes include altered biomechanical loading patterns following meniscectomy as well as global processes within the knee.
\end{abstract}

Keywords Knee $\cdot$ Meniscectomy $\cdot$ Patellofemoral joint $\cdot$ Tibiofemoral joint $\cdot$ Osteoarthritis $\cdot$ Long-term follow-up

\section{Introduction}

The importance of patellofemoral joint (PFJ) osteoarthritis $(\mathrm{OA})$ in relation to disability has been studied before [1] It has been demonstrated that a standardised skyline view

Kendrick To

kendrick.to@doctors.org.uk

1 Department of Trauma and Orthopaedics, Royal Cornwall Hospital, Truro TR1 3LQ, UK

2 Department of Trauma and Orthopaedics, Guy's Hospital, London SE1 9RT, UK

3 Department of Trauma and Orthopaedics, St Mary's Hospital, London W2 1NY, UK

4 Division of Trauma and Orthopaedics, Department of Surgery, Addenbrooke's Hospital, University of Cambridge, Cambridge CB2 0QQ, UK

5 Department of Trauma and Orthopaedics, Aintree University Hospital, Liverpool L9 7AL, UK radiograph provides more information of the PFJ than a lateral view radiograph and has greater reproducibility [2-4]. Whilst a dedicated skyline view may be superior to a lateral view in depicting PFJ OA [5, 6], not all orthopaedic surgeons utilise this. Some studies suggest that up to $75 \%$ of clinicians opt not to use a skyline view in their assessment of knee pain [7].

It has been well documented that meniscectomy is a significant risk factor for tibiofemoral joint (TFJ) OA [8-10], but there are fewer studies demonstrating its effect on the PFJ [11]. Some studies have shown that PFJ OA occurs most frequently in the lateral facet, with some reporting a frequency of $89 \%$ [12-14]. One particular study sought to 
further investigate the effects of joint space narrowing (JSN) to $<5 \mathrm{~mm}$ in the lateral facet [15]. In this study, we focus on the lateral facet. Our group previously published the effects of open meniscectomy on TFJ OA and patient-related outcome measures (PROMs) at a mean 40-year follow-up [16]. In this study, we are using the same cohort to assess the effects of meniscectomy on PFJ OA. A purpose-built device was validated [11] and used (Fig. 1) to assess this cohort. The same method of standardising a weight-bearing skyline view as per the aforementioned study was utilised.

\section{Methods}

Under the auspices of the late Professor Iain Smillie, 313 adolescent patients underwent open total meniscectomy between 1960 and 1980. Of those patients, 100 who were confidently identified as having no other intra-articular knee pathology at the time of operation were reviewed at 17 years and 30 years post-operatively. Fifty-three patients were evaluated radiologically. This study represents the ongoing follow-up of the 53 patients at a mean of 40 years (33 to 50 years). Several patients were lost to the follow-up or excluded; five patients had died, seven had undergone a total knee replacement, six were lost to the follow-up, three

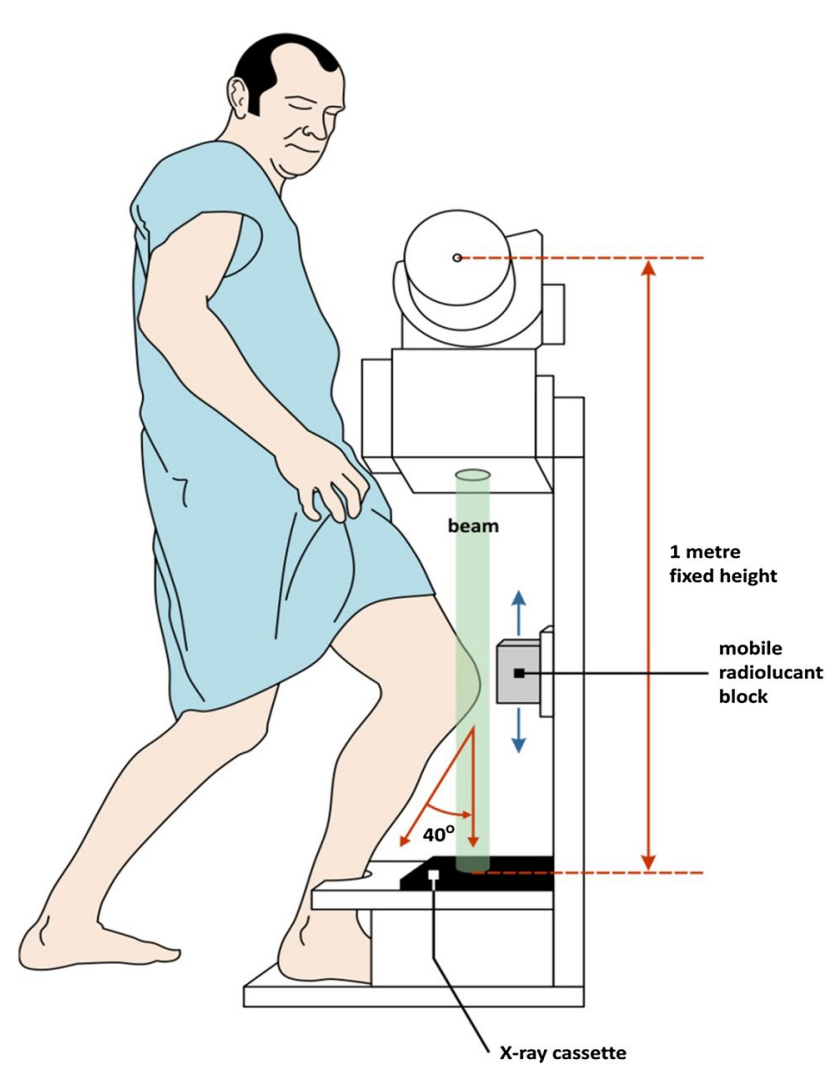

Fig. 1 Skyline view of knee obtained in $40^{\circ}$ of flexion declined to be assessed, and one was unable to attend due to multiple sclerosis. In addition, one patient who underwent lateral meniscectomy was unable to attend the clinic and completed the patient-related outcome measures (PROMs) questionnaires over the telephone; these data were excluded from subsequent statistical analysis. A total of 30 patients had objective and PROMs recorded at dedicated clinics. Eight patients had subsequently undergone interventions involving the contralateral knee after their 19th birthday; thus, the number of patients who had an intervention on one knee only at the time of review was 22 .

All patients were reviewed by one assessor (IP), the range of movement (ROM) in both knees was recorded using a long-levered goniometer, and anterior tibial translation (sagittal laxity) was recorded with the rolimeter device (DJO, Vista, California) by averaging three consecutive readings of each parameter. Patient-reported outcomes were assessed using the International Knee Documentation Committee (IKDC) score. The PFJ was evaluated by standardised weight-bearing images at approximately $40^{\circ}$ of flexion using a purpose-built device [11], as depicted in Fig. 1. The device projected a vertical beam of X-ray at a knee joint that was flexed to $40^{\circ}$ with the subject in a standing position. The standardised weight-bearing skyline views of both the operated and non-operated knees were assessed in a darkened room and from the set distance of $60 \mathrm{~cm}$ without magnification equipment and scored in terms of the presence of osteophytes and the joint space (in each facet) measured by a ruler in increments of $1 \mathrm{~mm}$. The KL score was utilised to quantify OA. Established OA was determined by the presence of osteophytes and its progression by JSN $[17,18]$.

\section{Statistical analysis}

The data were analysed with SPSS 17.0 (SPSS Inc., Chicago) statistical package, where a $p$ value of less than 0.05 was deemed to be statistically significant. A Shapiro-Wilk test was performed. Parametric data were analysed with a paired $t$ test, whilst nonparametric data were subject to Wilcoxon signed-rank and the Mann-Whitney $U$ tests. Kendall's tau coefficient was then applied to correlate statistical dependence.

\section{Results}

There was a significant difference between the operated and non-operated knees in the frequency of disease and the presence of osteophytes in the PFJ $(p=0.0015)$. The relative risk (RR) of developing radiologically diagnosed OA in the PFJ of operated versus non-operated knees was found to be 1.8 (95\% CI 1.13-2.96). 
Table 1 PFJ JSN and the presence of osteophytes between operated and non-operated knees

\begin{tabular}{llll}
\hline & $\begin{array}{l}\text { Operated } \\
\text { knee-mean } \\
( \pm \mathrm{SD})\end{array}$ & $\begin{array}{l}\text { Non-operated } \\
\text { knee-mean } \\
( \pm \mathrm{SD})\end{array}$ & $p$ value \\
\hline Medial joint space $(\mathrm{mm})$ & $4.32(2.88)$ & $5.32(2.06)$ & 0.037 \\
Lateral joint space $(\mathrm{mm})$ & $5.27(2.75)$ & $5.09(2.41)$ & 0.37 \\
Osteophytes & $1.41(1.14)$ & $0.45(0.8)$ & 0.0058 \\
\hline
\end{tabular}

Table 2 The presence of PFJ osteophytes and the type of meniscectomy performed

Presence of osteo- $\quad p$ value phytes

\begin{tabular}{lll}
\hline Medial meniscectomy & $4 / 7,57 \%$ & 0.288 \\
Lateral meniscectomy & $6 / 9,66 \%$ & \\
Medial and lateral meniscectomy & $6 / 6,100 \%$ & \\
\hline
\end{tabular}

A significant difference was seen between the operated knee and non-operated knee in terms of medial joint space; the space was measured to be $4.32 \mathrm{~mm}( \pm 2.88 \mathrm{~mm})$ and $5.32 \mathrm{~mm}( \pm 2.06 \mathrm{~mm})$, respectively (Table 1$)$. In contrast, no significant difference was observed in the lateral joint space. There was a significant difference in the presence of osteophytes between operated and non-operated knees when the knees were globally assessed $(1.41 \pm 1.14$ osteophytes and $0.45 \pm(0.8)$ osteophytes, respectively), with less osteophytes seen in non-operated knees.

When the presence of osteophytes in the PFJ was grouped into the type of meniscectomy, no significant difference was identified between lateral and medial meniscectomy (Table 2). Out of 22 patients, seven underwent medial, nine underwent lateral and six underwent both medial and lateral meniscectomy. Osteophytes were observed in four out of seven, six out of nine and six out of six subjects, respectively.

The lateral facet of the PFJ in the operated knee was analysed, and mean differences in KL, IKDC and ROM results in the TFJ were recorded. A comparison was made between the PFJ with $<5 \mathrm{~mm}$ lateral facet joint space and PFJ with lateral joint space $>5 \mathrm{~mm}$ (Table 3 ).

The results show an association between open total meniscectomy and PFJ OA at 40 years following surgery.
There was also an association between patellofemoral joint space narrowing in the lateral facet and TFJ OA. Possible causes include altered biomechanical loading patterns following meniscectomy as well as global processes within the knee.

\section{Discussion}

Evaluating patellofemoral joint PFJ OA can be challenging. To appropriately assess osteoarthritic change, we must bear basic principles in mind [17, 18]. For example, one scoring system, as proposed by Jones et al. [2], does not take joint space narrowing into consideration. Another study attempted to clarify the specificity of JSN as a measure of MRI-determined cartilaginous and osteoarthritic defects. A cut-off value of $5 \mathrm{~mm}$, i.e. if joint space in either the lateral or medial facets of the PFJ was less than $5 \mathrm{~mm}$, was found to have a high specificity for MR-detected cartilage defects. In addition, a separate study by the same group $[4,15]$ demonstrated that a PFJ with joint space of $<5 \mathrm{~mm}$ with the concomitant presence of osteophytes had sensitivity value and a positive predictive value of $90 \%$ and $95 \%$, respectively, for MR-detected cartilage defects. It was shown that this sensitivity was reduced if the joint space was greater than $5 \mathrm{~mm}$. Therefore, it is apparent that JSN to be less than $5 \mathrm{~mm}$, with the presence of osteophytes, is diagnostic of OA in the PFJ.

In our study, a significant difference was observed between the operated and non-operated knees in terms of osteophyte presence and JSN. This indicates a correlation between meniscectomised knees and the development of osteoarthritis was illustrated by an observed relative risk of 1.8 (95\% CI 1.13-2.96). In addition, there was no significant difference between the amount of joint space in the medial and lateral facets of the operated knees. However, the amount of lateral joint space demonstrated a significant effect on all measured patient outcomes. In addition, our analysis demonstrates a link between TFJ OA and PFJ OA following meniscectomy, as seen in the high KL score for the TFJ in operated knees with PFJ joint space of $<5 \mathrm{~mm}$. There may be several explanations to this finding. Knee OA in general has been shown to be associated with hand OA suggesting that osteoarthritis could be a genetic, systemic as well as localised disease [19]. Activation of cytokine and protease cascades which act globally within the affected
Table 3 Lateral PFJ JSN and its association with TFJ parameters

\begin{tabular}{lllc}
\hline & $\begin{array}{l}\text { PFJ lateral facet joint } \\
\text { space }<5 \mathrm{~mm}\end{array}$ & $\begin{array}{l}\text { PFJ lateral facet joint } \\
\text { space }>5 \mathrm{~mm}\end{array}$ & $p$ value \\
\hline TFJ KL score $($ mean $\pm 2 x S D)$ & $3.43(2.36-4.50)$ & $2.60(0.003-5.20)$ & 0.013 \\
IKDC (mean $\pm 2 x S D)$ & $54.7(41.4-68.0)$ & $65.8(43.1-88.5)$ & $<0.05$ \\
ROM (mean $\pm 2 x S D)$ & $118(89-147)$ & $129(110-147)$ & $<0.00001$ \\
\hline
\end{tabular}


joint as well as systemically [20] could be one explanation, whilst altered biomechanical loading patterns post-meniscectomy could be another explanation [21]. Generalised OA in the post-meniscectomy knee could result in quadriceps weakness through disuse and may increase the risk of PFJ $\mathrm{OA}$ as well as TFJ OA.

\section{Conclusions}

To our knowledge, this is the only study of osteoarthritis in the patellofemoral joint following meniscectomy that extends to a 40-year follow-up period. Our results suggest that meniscectomy increases the likelihood of subsequent patellofemoral joint osteoarthritis. This is associated with poorer functional outcomes. The underlying process appears to affect multiple parts of the knee joint. It is therefore important, when performing meniscectomies in young patients, to consider the implications of surgery on the longterm function of the knee joint. Consequently, there may be benefit in conducting long-term follow-up assessment in these patients.

Author contributions IP was the lead author involved in inception of and execution of study, data collection and analysis. (This study was part of his Medical Doctorate Thesis), WN and AA assisted in radiographic assessment and in conducting literature search. KT contributed to writing the manuscript under the supervision of WK. MM conducted follow-up of the study cohort at 30 years. All authors conducted data analysis and proofreading of the manuscript prior to submission.

\section{Compliance with ethical standards}

Conflict of interest The authors declare that they have no conflict of interest.

Open Access This article is distributed under the terms of the Creative Commons Attribution 4.0 International License (http://creativeco mmons.org/licenses/by/4.0/), which permits unrestricted use, distribution, and reproduction in any medium, provided you give appropriate credit to the original author(s) and the source, provide a link to the Creative Commons license, and indicate if changes were made.

\section{References}

1. McAlindon TE, Snow S et al (1992) Radiographic patterns of osteoarthritis of the knee joint in the community: the importance of the patellofemoral joint. Ann Rheum Dis 51(7):844-849

2. Jones AC, Ledingham J et al (1993) Radiographic assessment of patellofemoral osteoarthritis. Ann Rheum Dis 52(9):655-658

3. Cicuttini FM, Baker J et al (1996) Choosing the best method for radiological assessment of patellofemoral osteoarthritis. Ann Rheum Dis 55(2):134-136
4. Boegard T, Rudling O et al (1998) Joint-space width in the axial view of the patello-femoral joint. Definitions and comparison with MR imaging. Acta Radiol 39(1):24-31

5. Lanyon P, Jones A et al (1996) Assessing progression of patellofemoral osteoarthritis: a comparison between two radiographic methods. Ann Rheum Dis 55(12):875-879

6. Davies AP, Vince AS et al (2002) The radiologic prevalence of patellofemoral osteoarthritis. Clin Orthop Relat Res 402:206-212

7. Vince AS, Singhania AK et al (2000) What knee X-rays do we need? A survey of orthopaedic surgeons in the United Kingdom. Knee 7(2):101-104

8. Fairbank TJ (1948) Knee joint changes after meniscectomy. J Bone Joint Surg Br 30B(4):664-670

9. Jorgensen U, Sonne-Holm S et al (1987) Long-term follow-up of meniscectomy in athletes. A prospective longitudinal study. J Bone Joint Surg Br 69(1):80-83

10. Roos EM, Roos HP et al (1998) Knee Injury and Osteoarthritis Outcome Score (KOOS)—development of a self-administered outcome measure. J Orthop Sports Phys Ther 28(2):88-96

11. Englund M, Lohmander LS (2005) Patellofemoral osteoarthritis coexistent with tibiofemoral osteoarthritis in a meniscectomy population. Ann Rheum Dis 64(12):1721-1726

12. Casscells SW (1978) Gross pathological changes in the knee joint of the aged individual: a study of 300 cases. Clin Orthop Relat Res 132:225-232

13. Ficat $P$ (1978) The syndrome of lateral hyperpressure of the patella. Acta Orthop Belg 44(1):65-76

14. Iwano T, Kurosawa $\mathrm{H}$ et al (1990) Roentgenographic and clinical findings of patellofemoral osteoarthrosis. With special reference to its relationship to femorotibial osteoarthrosis and etiologic factors. Clin Orthop Relat Res 252:190-197

15. Boegard T, Rudling O et al (1998) Correlation between radiographically diagnosed osteophytes and magnetic resonance detected cartilage defects in the tibiofemoral joint. Ann Rheum Dis 57(7):401-407

16. Pengas IP, Assiotis A, Nash W, Hatcher J, Banks J, McNicholas MJ (2012) Total meniscectomy in adolescents: a 40-year follow-up. J Bone Joint Surg Br 94:1649-1654. https://doi. org/10.1302/0301-620X.94B12.30562

17. Altman R, Asch E et al (1986) Development of criteria for the classification and reporting of osteoarthritis. Classification of osteoarthritis of the knee. Diagnostic and Therapeutic Criteria Committee of the American Rheumatism Association. Arthritis Rheum 29(8):1039-1049

18. Altman RD, Fries JF et al (1987) Radiographic assessment of progression in osteoarthritis. Arthritis Rheum 30(11):1214-1225

19. Englund M, Paradowski PT et al (2004) Association of radiographic hand osteoarthritis with radiographic knee osteoarthritis after meniscectomy. Arthritis Rheum 50(2):469-475

20. Sandy JD (2003) Proteolytic degradation of normal and osteoarthritic cartilage matrix. In: Brandt KD, Doherty M, Lohmander LS (eds) Osteoarthritis. Oxford University Press, Oxford, pp 82-92

21. Elahi S, Cahue $S$ et al (2000) The association between varus-valgus alignment and patellofemoral osteoarthritis. Arthritis Rheum 43(8):1874-1880

Publisher's Note Springer Nature remains neutral with regard to jurisdictional claims in published maps and institutional affiliations. 